\title{
COSPAR2006 PEDAS1-0011-06
}

\section{Instability of the Present LEO Satellite Populations}

\author{
J.-C. Liou ${ }^{1}$ and N. L. Johnson ${ }^{2}$ \\ ${ }^{1}$ ESCG/ERC, Mail Code JE104, 2224 Bay Area Blvd., Box 7, Houston, TX 77058, USA, \\ jer-chyi.liou-1@nasa.gov \\ ${ }^{2}$ Orbital Debris Program Office, NASA Johnson Space Center, Houston, TX 77058, USA, \\ nicholas.l.johnson@nasa.gov
}

\begin{abstract}
Several studies conducted during 1991-2001 demonstrated, with some assumed launch rates, the future unintended growth potential of the Earth satellite population, resulting from random, accidental collisions among resident space objects. In some low Earth orbit (LEO) altitude regimes where the number density of satellites is above a critical spatial density, the production rate of new breakup debris due to collisions would exceed the loss of objects due to orbital decay.

A new study has been conducted in the Orbital Debris Program Office at the NASA Lyndon B. Johnson Space Center, using higher fidelity models to evaluate the current debris environment. The study assumed no satellites were launched after December 2005. A total of 150 Monte Carlo runs were carried out and analyzed. Each Monte Carlo run simulated the current debris environment and projected it 200 years into the future. The results indicate that the LEO debris environment has reached a point such that even if no further space launches were conducted, the Earth satellite population would remain relatively constant for only the next 50 years or so. Beyond that, the debris population would begin to increase noticeably, due to the production of collisional debris. Detailed analysis shows that this growth is primarily driven by high collision activities around 900 to $1000 \mathrm{~km}$ altitude - the region which has a very high concentration of debris at present.

In reality, the satellite population growth in LEO will undoubtedly be worse than this study indicates, since spacecraft and their orbital stages will continue to be launched into space. Postmission disposal of vehicles (e.g., limiting postmission orbital lifetimes to less than 25 years) will help, but will be insufficient to constrain the Earth satellite population. To preserve better the near-Earth environment for future space activities, it might be necessary to remove existing large and massive objects from regions where high collision activities are expected.
\end{abstract}

\section{Introduction}

Since the launch of Sputnik 1, space activities have created an orbital debris environment that poses increasing impact risks to existing space systems, including human space flight and robotic missions. Although the Space Shuttle and International Space Station normally operate in the relatively pristine regime between 200 and $400 \mathrm{~km}$ above the Earth, their large cross-sectional areas yield collision rates comparable to their smaller robotic cousins in the more densely populated, higher altitude regions. Three accidental collisions between cataloged satellites during the period from late 1991 to early 2005 have already been documented (Anon 2005), although fortunately none resulted in the creation of large, trackable debris clouds. Currently, more than 
9000 Earth orbiting man-made objects, with a combined mass exceeding 5 million kilograms, are tracked by the US Space Surveillance Network (SSN) and maintained in the US satellite catalog (Anon 2006).

As the satellite population in the low Earth orbit (LEO, 200 to $2000 \mathrm{~km}$ altitude) region increases, the probabilities of mutual collisions among orbiting objects also increase. High collision activities may produce more fragments than those removed by drag in some altitude regimes. This off-balance may lead to a collision cascade such that collision fragments induce more collisions in the environment (Kessler and Cour-Palais 1978; Eichler and Rex 1990). The concept of "critical density" was pioneered by Kessler (1991) to characterize this uncontrollable population growth. Additional studies, based on analytical, semi-analytical, and numerical approaches, were carried out by various groups to analyze this phenomenon (Su 1993; Rossi et al. 1994; Anselmo et al. 1997; Kessler 2000; Kessler and Anz-Meador 2001; Krisko et al. 2001a). These studies adopted different model assumptions, initial conditions and future launch rates. Some of them even included different postmission disposal measures in the simulations. They all came to a similar conclusion that, indeed, the LEO debris populations at some altitudes would be unstable. Collisions would take over as the dominant debris generation mechanism, and the debris generated would feed back to the environment and induce more collisions.

The objective of this paper is to evaluate the instability of the current LEO debris environment with a different approach. In addition to using a recently developed high fidelity debris evolutionary model, with updated debris populations as initial conditions, this new study also made a key assumption - it assumed no new launches were conducted after December 2005. This assumption eliminated several major uncertainties in debris environment projection, such as future launch frequency, types of satellites launched, and their physical and orbital specifications. Although "no future launches" is an unrealistic assumption, it does provide a reliable bottom line assessment of the current LEO debris environment. This environmental best case scenario can also serve as a benchmark to evaluate whether or not current mitigation measures are sufficient to control the growth of future debris populations. A short summary of a similar study, using the historical populations through the end of 2004, has been published (Liou and Johnson 2006). This paper provides additional details on the analysis with the historical populations through the end of 2005, and with more Monte Carlo simulations.

\section{Model Description}

NASA's orbital debris evolutionary model LEGEND (LEO-to-GEO Environment Debris model) is a three-dimensional model. It is capable of simulating the historical and future debris populations in the near-Earth environment (Liou et al. 2004; Liou 2005). The historical simulation in LEGEND covers the period from 1957 to the end of 2005 for this study. The model adopts a deterministic approach to mimic the known historical populations. Launched rocket bodies, spacecraft, and mission-related debris (rings, bolts, etc.) are added to the simulated environment based on a comprehensive NASA Orbital Debris Program Office internal database. Known historical breakup events (Johnson et al. 2004) are reproduced, and fragments down to $1 \mathrm{~mm}$ in size are created with the NASA Standard Breakup Model, which describes the size, area-to-mass, and velocity distributions of the breakup fragments (Johnson et al. 2001). All objects are propagated forward in time while decayed objects are removed from the environment immediately. Perturbations included in the orbit propagation are Earth's $\mathrm{J}_{2}, \mathrm{~J}_{3}, \mathrm{~J}_{4}$, solar-lunar gravitational perturbations, atmospheric drag, solar radiation pressure, and Earth's shadow effects. Historical daily solar flux F10.7 values are combined with the J77 atmospheric model for the drag calculation 
(Jacchia 1977). The simulation program outputs the orbital elements and other physical properties of the objects at the end of each year for post-processing analysis.

The future projection component of LEGEND covers 200 years from the end of the historical simulation. As stated previously, this study assumed no rocket bodies and spacecraft were launched after December 2005. No future disposal maneuvers were allowed for existing spacecraft, few of which currently have such a capability. Orbit propagation is handled in a way identical to the historical simulation. The solar flux F10.7 values used in the projection period have two components: a short-term projection (2006-2007, obtained from NOAA Space Environment Center) and a long-term projection (2008-2104). The long-term F10.7 projection is a repeat of a sixth-order sine and cosine functional fit to Solar Cycles 18 to 23 (Whitlock 2006). A simple smoothing function is used to interpolate the two solar flux components during the short- to longterm transition.

Explosion probabilities of future rocket bodies and spacecraft are based on an analysis of launch history and recent explosions. The explosion probability of each explodable vehicle is set to zero after 10 years of on-orbit lifetime. Vehicles with a history of explosion, but which have had the breakup causes fixed, are not included in future explosion consideration. Collision probabilities among objects are estimated with a fast pair-wise comparison algorithm in the projection component (Liou 2005). Only objects $10 \mathrm{~cm}$ and larger are considered for potential collisions. This size threshold is historically the detection limit of the SSN sensors, and more than $95 \%$ of the debris population mass is in objects $10 \mathrm{~cm}$ and larger. Fragments down to $1 \mathrm{~mm}$ in size are generated based on the NASA Standard Breakup Model, and are added to the simulated environment afterward. Each breakup fragment is assigned a unique identification character to track its origin: explosion, intact-intact collision, intact-fragment collision, or fragment-fragment collision.

The LEGEND future projection adopts a Monte Carlo (MC) approach to simulate potential future on-orbit explosions and collisions. The procedure of this MC approach is simple and straightforward. Within a given projection time step, 5 days in this study, once the explosion probability is estimated for an intact object, a random number is drawn and compared with the probability to determine if an explosion would occur. A similar procedure is applied to collisions for each pair of target and projectile involved within the same time step. Parent objects are removed from the environment once fragments are generated. Due to the nature of the MC processes, multiple projection runs must be performed and analyzed before reliable and meaningful conclusions can be drawn from the outcome. A statistical study of LEGEND, based on the standard bootstrap method, indicates that the mean from $100 \mathrm{MC}$ runs leads to a standard error of the mean, at the end of a 100-year projection, on the order of $1 \%$ or less (Liou 2006). Therefore, averages from $150 \mathrm{MC}$ runs presented in this paper should be statistically sound and reliable.

\section{LEGEND Predictions}

The simulated $10 \mathrm{~cm}$ and larger debris populations in LEO, between 1957 and the end of the 200-year future projection, are shown in Figure 1. The historical parts of the curves are from a single deterministic run while the projection parts of the curves are averages from $150 \mathrm{MC}$ runs. The effective number is defined as the fractional time, per orbital period, an object spends between 200 and $2000 \mathrm{~km}$ altitude. From the breakdown of the three sub-populations, it is obvious that collision fragments replace other decaying debris through the next 50 years or so, keeping the total LEO population approximately constant. Beyond that, however, the rate of the natural reentry of 
debris declines (most remaining satellites are long-lived objects - with low area-to-mass ratio, and/or high altitude), leading to a net increase in the overall satellite population due to collisions.

An average of 17.1 collisions (10.3 catastrophic, 6.8 non-catastrophic) would be expected in the next 200 years based on the "no new launches" scenario (Table 1). A catastrophic collision occurs when the ratio of impact kinetic energy to target mass exceeds $40 \mathrm{~J} / \mathrm{g}$ (Johnson et al. 2001). The outcome of a catastrophic collision is the total fragmentation of the target object, whereas a non-catastrophic collision only results in minor physical damage to the target and generates a small amount of debris that has negligible contribution to the population growth. In general two massive debris clouds are generated from every intact-intact catastrophic collision while only one massive debris cloud is generated from an intact-fragment catastrophic collision. Since there are twice as many intact-fragment catastrophic collisions as there are intact-intact catastrophic collisions (see Table 1), these two types of collisions contribute more or less equally to the debris population growth over the next 200 years.

Based on Table 1, the average LEO catastrophic collision frequency is $5.2 \%$ per year, through 2205, for this "no new launches" scenario. Due to the nature of the Monte Carlo simulations, therefore, on average one out of every 19 MC runs would predict a catastrophic collision in the first future projection year. This is why collision fragments (averaged over $150 \mathrm{MC}$ runs) were created from the very beginning of the future projection simulation (when the MC collision algorithm was switched on), as shown in Figure 1. The immediate increase in collision fragments does not mean LEGEND is predicting one or more collisions in 2006. Rather, it is only predicting a fraction of a catastrophic collision (again, a result of averaging over $150 \mathrm{MC}$ runs) in the first year. When the cumulative number of catastrophic collisions is plotted against time, as shown in Figure 2, it is clear that what LEGEND predicts is one catastrophic collision by 2028. Another interesting point about the figure is the trend increases almost linearly with time in the next 200 years, although there is a slightly higher increase after 50 years (compared with the dotted reference line). This is also consistent with the population growth after 2055, as shown in Figure 1.

Another consequence of displaying the averages is the smoothness of the curves. In reality, the population growth would be quite choppy with a sudden upward jump accompanying every breakup. Depending on the altitude and/or perigee of the fragment cloud, the new debris may decay rather rapidly or may decay over a much longer period of time. This is illustrated in Figure 3 where individual population growths from the first $50 \mathrm{MC}$ runs are shown. The distribution of the LEO satellite populations (objects $10 \mathrm{~cm}$ and larger) at the end of the 200-year projection from all 150 MC runs is shown in Figure 4. The LEO satellite population (objects $10 \mathrm{~cm}$ and larger) at the beginning of the projection ( 1 January, 2006) is 7667, as indicated by arrow 1 . The median and mean of the distribution are very close to each other (10,686 and 11,149, respectively), and are indicated by arrows 2 and 3 in the same figure. Individually, each one of the $150 \mathrm{MC}$ runs represents an equally probable outcome of the "no new launches" scenario. Collectively, 23 out of the 150 MC runs ( 15\%) predict a population decrease in 200 years while the remaining 127 runs ( $85 \%)$ predict a population growth in 200 years even without new launches.

As expected, the distribution of predicted catastrophic collision activities is non-uniform throughout LEO, with various impact speeds (Figure 5). Most collisions are in the hypervelocity impact regime above $\sim 2 \mathrm{~km} / \mathrm{sec}$. The concentration around $14 \mathrm{~km} / \mathrm{sec}$ comes from near head-on collisions between two groups of satellites with inclinations around $83^{\circ}$ and $99^{\circ}$. The highest concentration in altitude is around 900 to $1000 \mathrm{~km}$ altitude where the object spatial density is high (Figure 6). About $60 \%$ of all catastrophic collisions occur in this region, leading to a major 
population increase over time (Figure 7). There is a factor of more than 3 increase for objects 10 $\mathrm{cm}$ and larger in 200 years (Figure 8). This will lead to approximately a factor of 10 increase in collision probability. The high collision activities in this region are primarily responsible for the LEO population growth shown in Figure 1. Several factors contribute to the high collision activities in this region: higher spatial density, larger rocket bodies and spacecraft, two major inclination concentrations around $83^{\circ}$ and $99^{\circ}$ (leading to more near head-on collisions), and longer orbit decay time (compared with lower altitude regions) in this region.

\section{Discussion}

Has the current debris population in the LEO region reached the point where the environment is unstable and collisions will force an uncontrollable population growth in the foreseeable future? Based on this "no new launches" scenario, collisions will continue to occur in LEO over the next 200 years, primarily driven by the high collision activities in the region between 900 and $1000 \mathrm{~km}$ altitude. This trend, of course, will not last forever as long as no new launches are added to the environment. Over time (millennia or longer) the collision cascade process will deplete most massive objects in the environment and lead to a permanent population decrease at the end.

The "no new launches" assumption adopted for the study is, of course, not practical. However, it does serve as a good benchmark to assess the current debris environment. In reality, the LEO population growth will be greater than that shown in this paper, as spacecraft and their orbital stages continue to be launched into space. How much greater it will be in 200 years is difficult to predict, due to major uncertainties in future launches, although one can make reasonable assumptions to bound the problem (e.g., Krisko 2004)

Commonly adopted mitigation measures, such as limiting postmission orbital lifetimes of satellites to less than 25 years, will slow down the population growth (e.g., Krisko et al. 2001b; Walker et al. 2001; Liou and Johnson 2005). However, these measures will be insufficient to constrain the Earth satellite population. Only remediation of the near-Earth environment, i.e., removing existing large and massive objects from orbit, will likely prevent the undesirable effects predicted in the present study.

\section{References}

Anon, Accidental collisions of cataloged satellites identified, Orbital Debris Quarterly News, 9, 2, p1, NASA Johnson Space Center, Houston, Texas (2005).

Anon, Monthly number of cataloged objects in Earth orbit by object type, Orbital Debris Quarterly News, 10, 2, p10, NASA Johnson Space Center, Houston, Texas (2006).

Anselmo, L., Cordelli, A., Farinella, P., Pardini., C., Rossi, A. Modeling the evolution of the space debris population: recent research work in Pisa, ESA SP-393, 339-344, ESOC, Darmstadt, Germany (1997).

Eichler, P. Rex, D. Chain reaction of debris generation by collisions in space - A final threat to spaceflight, Acta. Astronautica, 22, 381-387 (1990).

Jacchia, L.G., Thermospheric Temperature, Density, and Composition: New model, Smithsonian Special Report SAO 375, Cambridge, Massachusetts (1977).

Johnson, N.L., Krisko, P.H., Liou, J.-C., Anz-Meador, P.D. NASA's new breakup model of EVOLVE 4.0, Adv. Space Res., 28, 9, 1377-1384 (2001). 
Johnson, N.L., Whitlock, D.O., Anz-Meador, P.D., Cizek, E.M., Portman, S.A. History of On-Orbit Satellite Fragmentations, 13th Edition. JSC-62530, NASA Johnson Space Center, Houston, Texas (2004).

Kessler, D.J. Collisional cascading: The limits of population growth in low Earth orbit, Adv. Space Res., 11, 12, 63-66 (1991).

Kessler, D.J. Critical Density of Spacecraft in Low Earth Orbit, NASA JSC-28949, NASA Johnson Space Center, Houston, Texas (2000).

Kessler, D.J. and Cour-Palais, B.G. Collision frequency of artificial satellites: The creation of a debris belt, JGR, 83, A6, 2637-2646 (1978).

Kessler, D.J. and Anz-Meador, P.D. Critical number of spacecraft in Low Earth Orbit: Using satellite fragmentation data to evaluate the stability of the orbital debris environment, ESA SP473, 265-272, ESOC, Darmstadt, Germany (2001).

Krisko, P.H. EVOLVE historical and projected orbital debris test environments, Adv. Space Res., 34, 5, 975-980 (2004).

Krisko, P.H., Opiela, J.N., Kessler, D.J. The critical density theory in LEO as analyzed by EVOLVE 4.0, ESA SP-473, 273-278, ESOC, Darmstadt, Germany (2001a).

Krisko, P.H., Johnson, N.L., Opiela, J.N. EVOLVE 4.0 orbital debris mitigation studies, Adv. Space Res., 28, 9, 1385-1390 (2001b).

Liou, J.-C., Hall, D.T., Krisko, P.H., Opiela, J.N. LEGEND - A three-dimensional LEO-to-GEO debris evolutionary model, Adv. Space Res., 34, 5, 981-986 (2004).

Liou, J.-C. Collision Activities in the future orbital debris environment. Adv. Space Res. (doi:10.1016/j.asr.2005.06.021), in press (2006).

Liou, J.-C., Johnson, N.L. A LEO Satellite postmission disposal study using LEGEND, Acta Astronautica, 57, 324-329 (2005).

Liou, J.-C. A statistical analysis on the future debris environment, Acta Astronautica, in press, (2006).

Liou, J.-C. and Johnson, N.L. Risks in space from orbiting debris, Science 311, 340-341 (2006).

Rossi, A., Cordelli, A., Farinella, P., Anselmo, L. Collisional evolution of the Earth's orbital debris cloud, JGR, 99, E11, 23195-23210 (1994).

Su, S.-Y. On runaway conditions of orbital debris environment, Adv. Space Res., 13, 8, 221-224 (1993).

Walker, R., Martin, C.E., Stokes, P.H., Wilkinson, J.E., Klinkrad, H. Analysis of the effectiveness of space debris mitigation measures using the DELTA model, Adv. Space Res., 28, 9, 14371445 (2001).

Whitlock, D. Modeling the effct of high solar activity on the orbital debris environment, Orbital Debris Quarterly News, 10, 2, p4, NASA Johnson Space Center, Houston, Texas (2006). 
Table 1. Future collisions, by object type, predicted by LEGEND. The numbers are averages from 150 Monte Carlo simulations assuming no new launches after December 2005.

\begin{tabular}{|c|c|c|}
\hline Object Type & $\begin{array}{c}\text { Number of collisions for the } \\
\text { next 200 years }\end{array}$ & $\begin{array}{c}\text { Catastrophic / } \\
\text { Non-catastrophic }\end{array}$ \\
\hline intact-intact & 4.9 & $4.8 / 0.1$ \\
\hline intact-fragment & 10.8 & $4.5 / 6.3$ \\
\hline fragment-fragment & 1.4 & $1.0 / 0.4$ \\
\hline Total & 17.1 & $10.3 / 6.8$ \\
\hline
\end{tabular}




\section{Figure Captions}

Figure 1: Effective number of LEO objects, $10 \mathrm{~cm}$ and larger, from the LEGEND simulations based on the "no new launches" assumption. The future projection parts of the curves (years 2006 to 2205) are averages from 150 Monte Carlo runs. Intacts are rocket bodies and spacecraft that have not experienced breakups.

Figure 2: Cumulative numbers of catastrophic collisions as a function of time. LEGEND predicts one catastrophic collision by 2028 even without any new launches. Comparison of the trend with a constant-slope reference line indicates that there is a slightly higher increase after about 50 years, consistent with the total population increase shown in Figure 1.

Figure 3: Individual population growths (effective number of LEO objects $10 \mathrm{~cm}$ and larger) from the first $50 \mathrm{MC}$ simulations. The sudden upward jumps are caused by breakups associated with each individual simulation.

Figure 4: LEO satellite populations (objects $10 \mathrm{~cm}$ and larger) at the end of 2205 from 150 different MC runs. The population at the beginning of 2006 is indicated by arrow 1 while the median and mean of the distribution are indicated by arrows 2 and 3, respectively.

Figure 5: Impact speed versus collision altitude for all LEO catastrophic collisions predicted from all 150 Monte Carlo runs for the next 200 years. Collisions are concentrated in high spatial density regions, and most of them are hypervelocity impacts.

Figure 6: Normalized distribution of predicted catastrophic collisions as a function of altitude. About $60 \%$ of them occur between 900 and $1000 \mathrm{~km}$ altitude.

Figure 7: Spatial density distributions, for objects $10 \mathrm{~cm}$ and larger, at the end of 2005 and 2205. The latter is the average of $150 \mathrm{MC}$ runs.

Figure 8: The population growth (10 cm and larger objects) between 900 and $1000 \mathrm{~km}$ altitude. The LEO population growth is primarily driven by the increase in this region. 
Figure 1

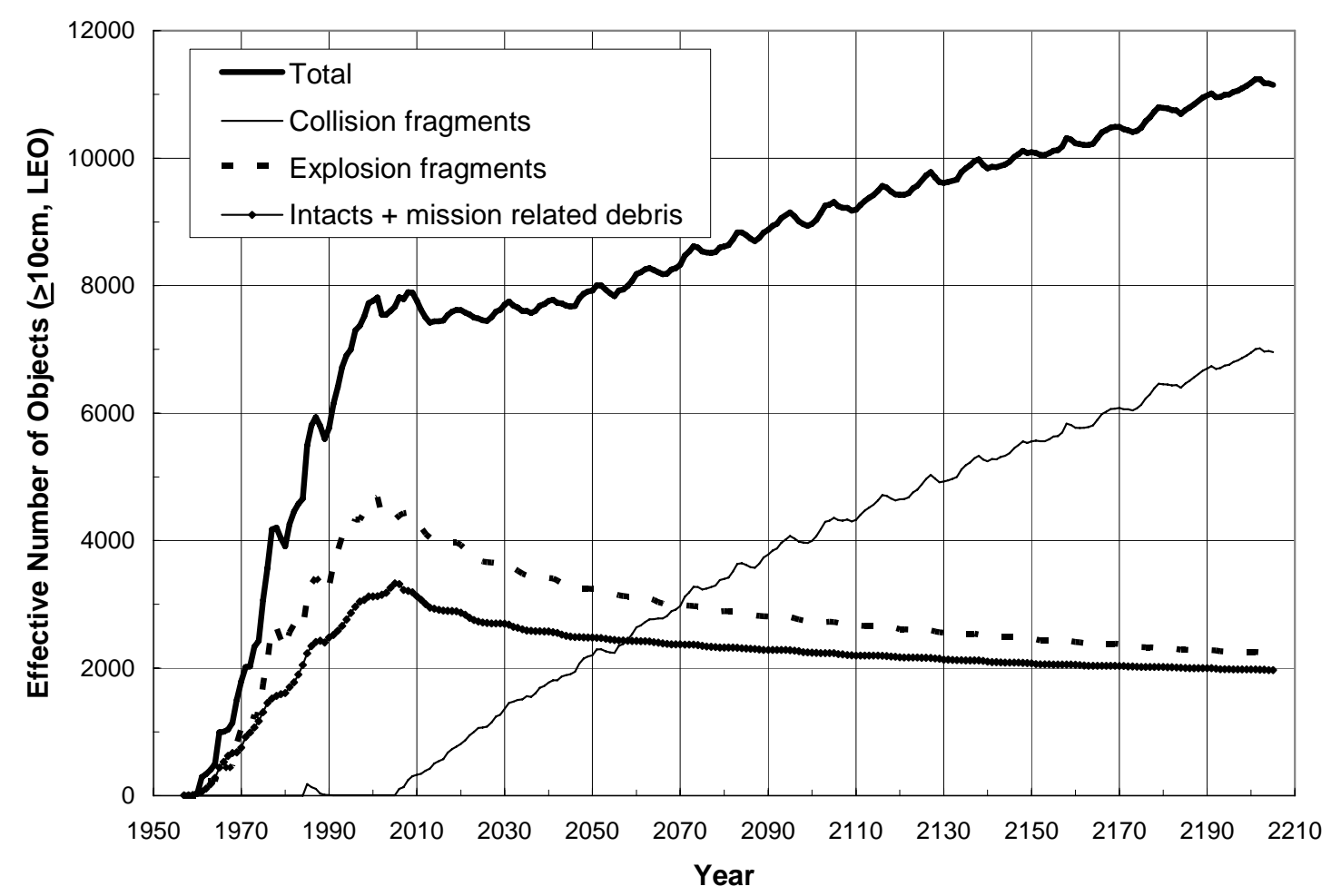


Figure 2

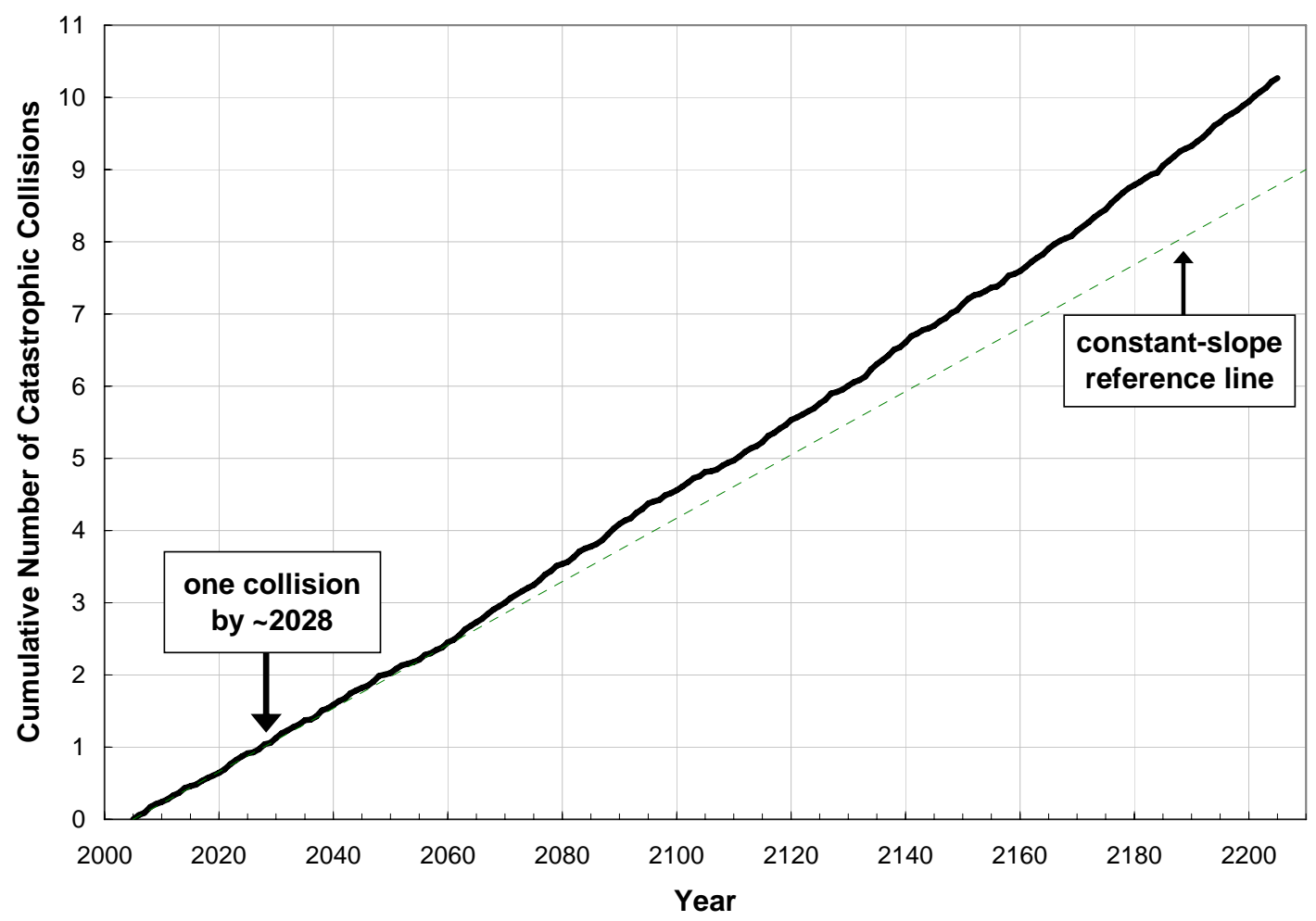


Figure 3

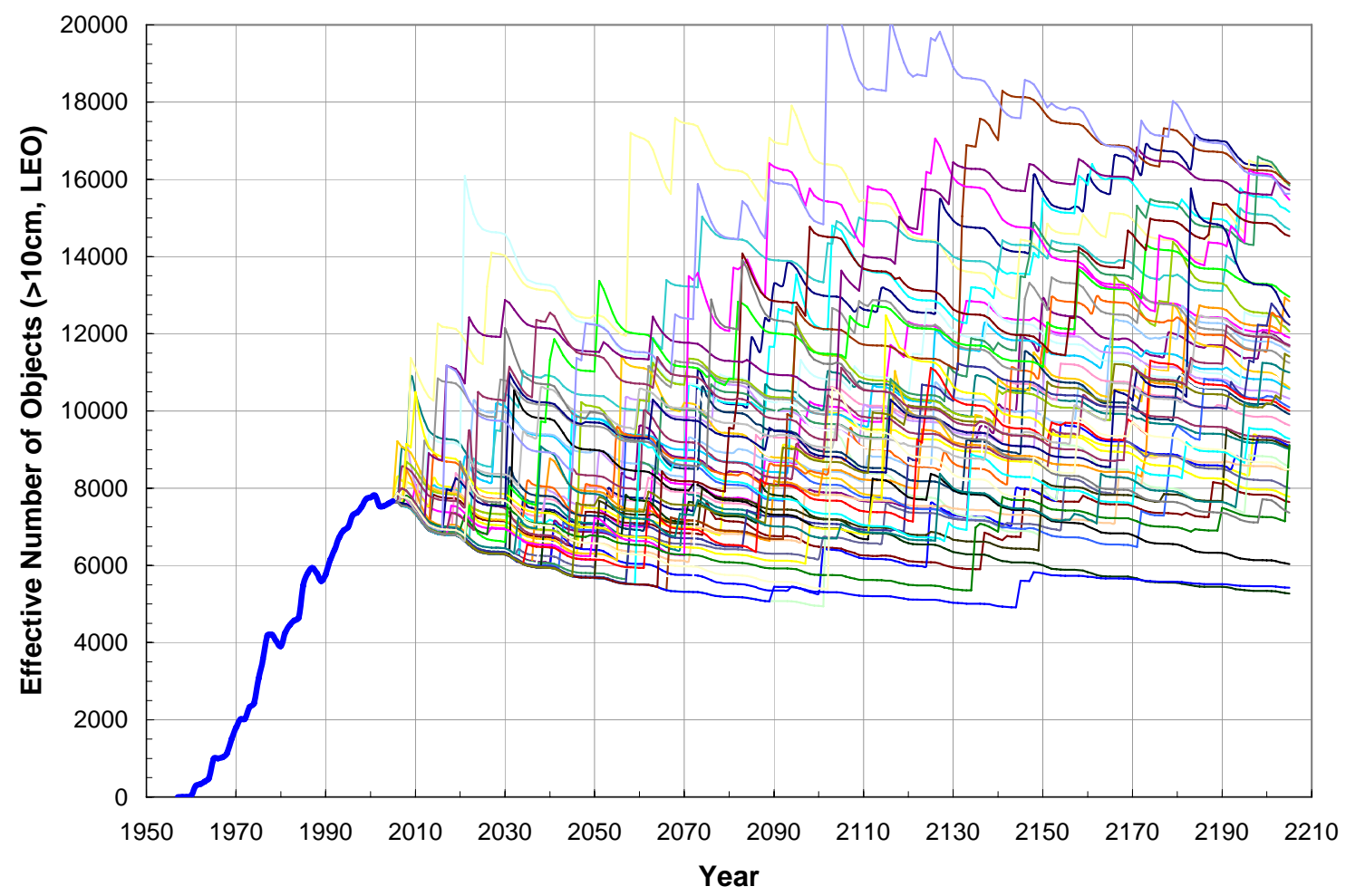


Figure 4

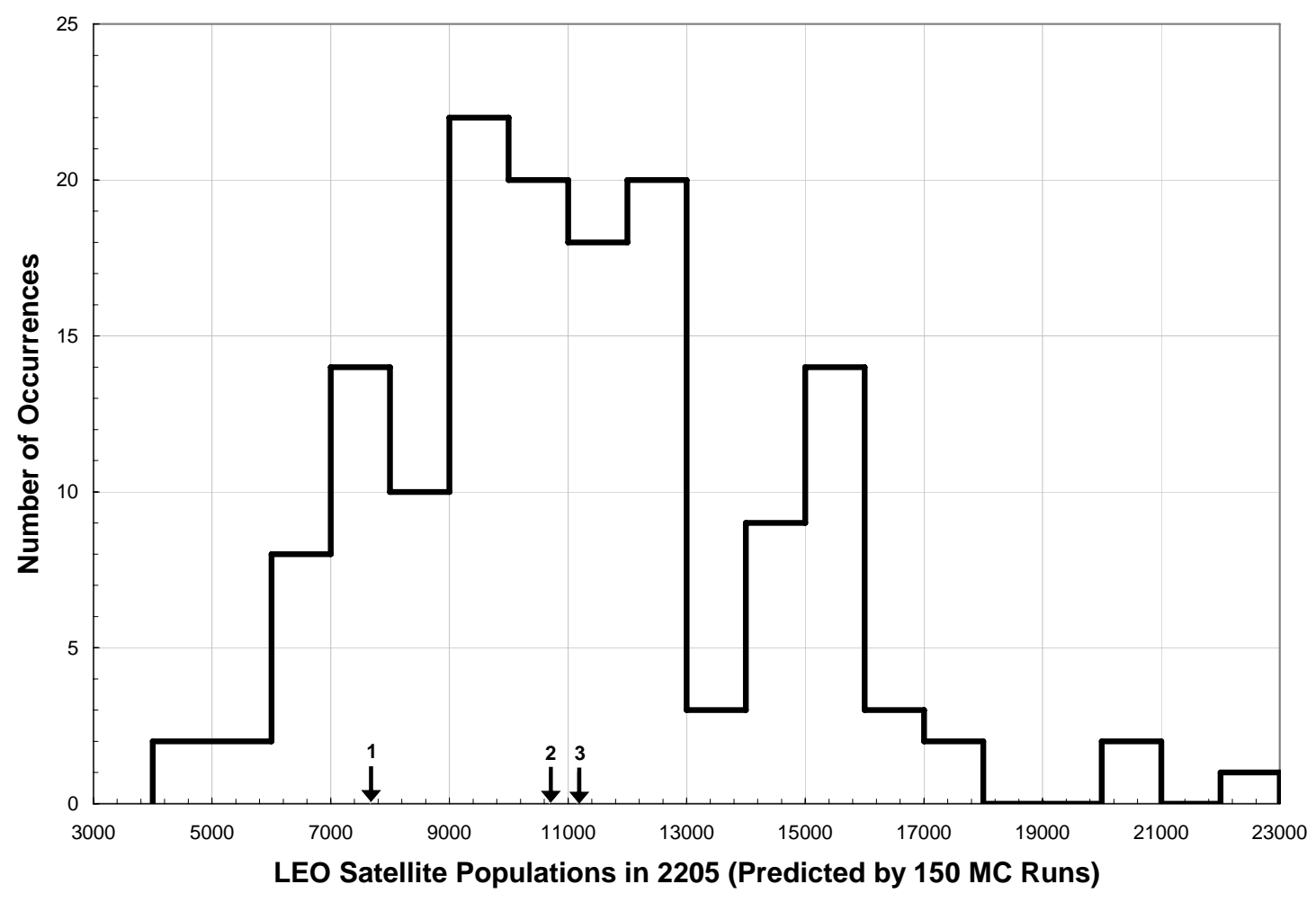


Figure 5

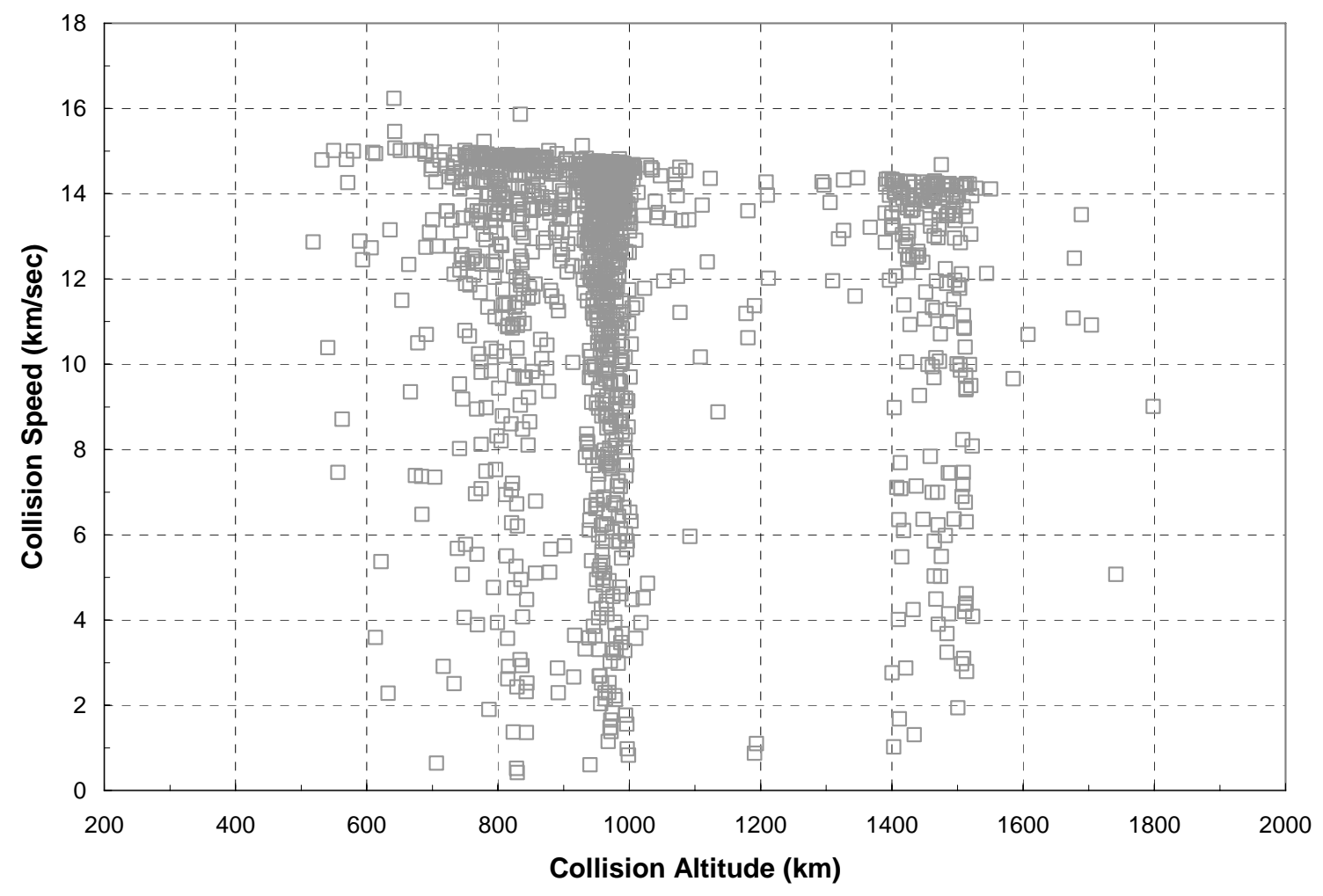


Figure 6

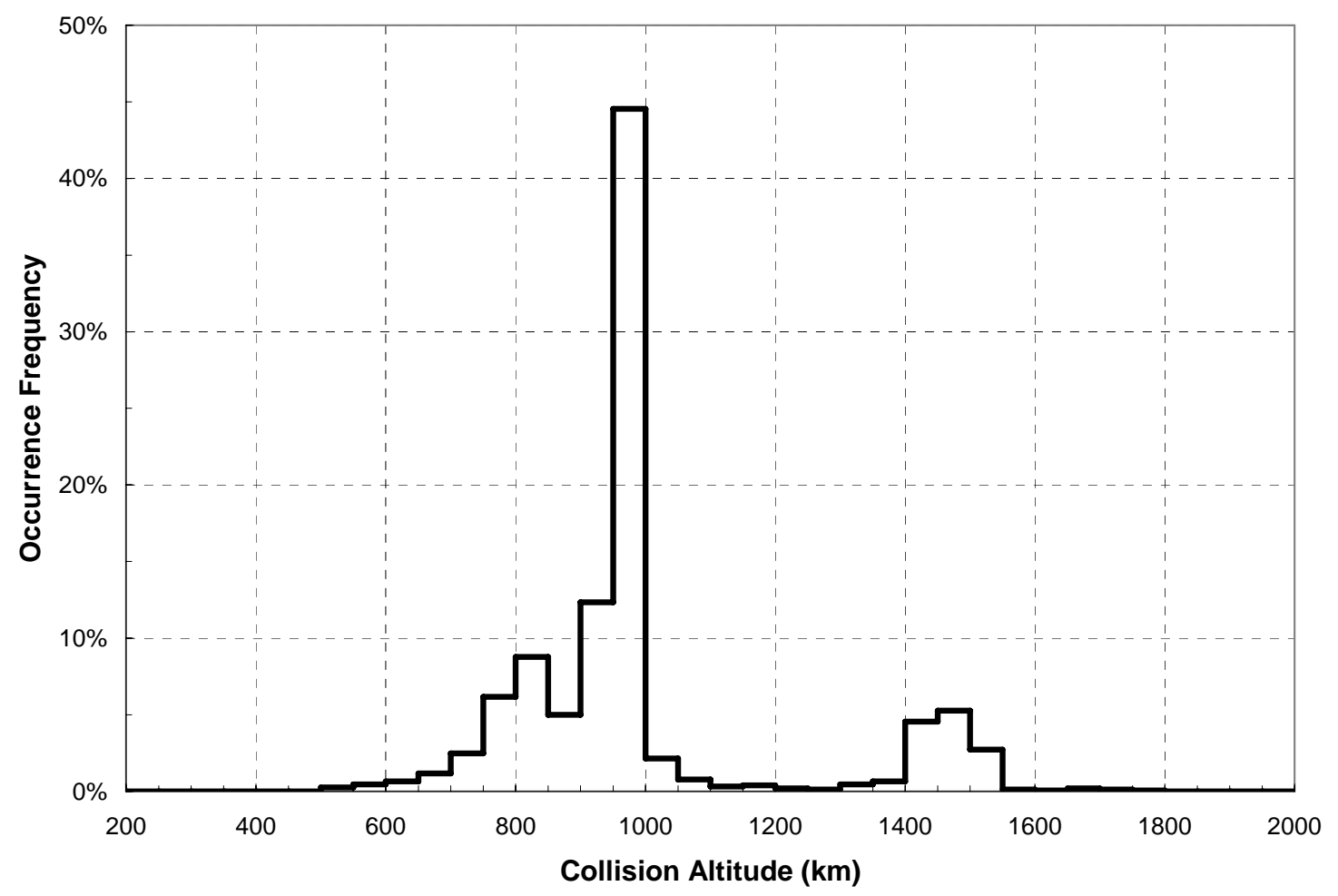


Figure 7

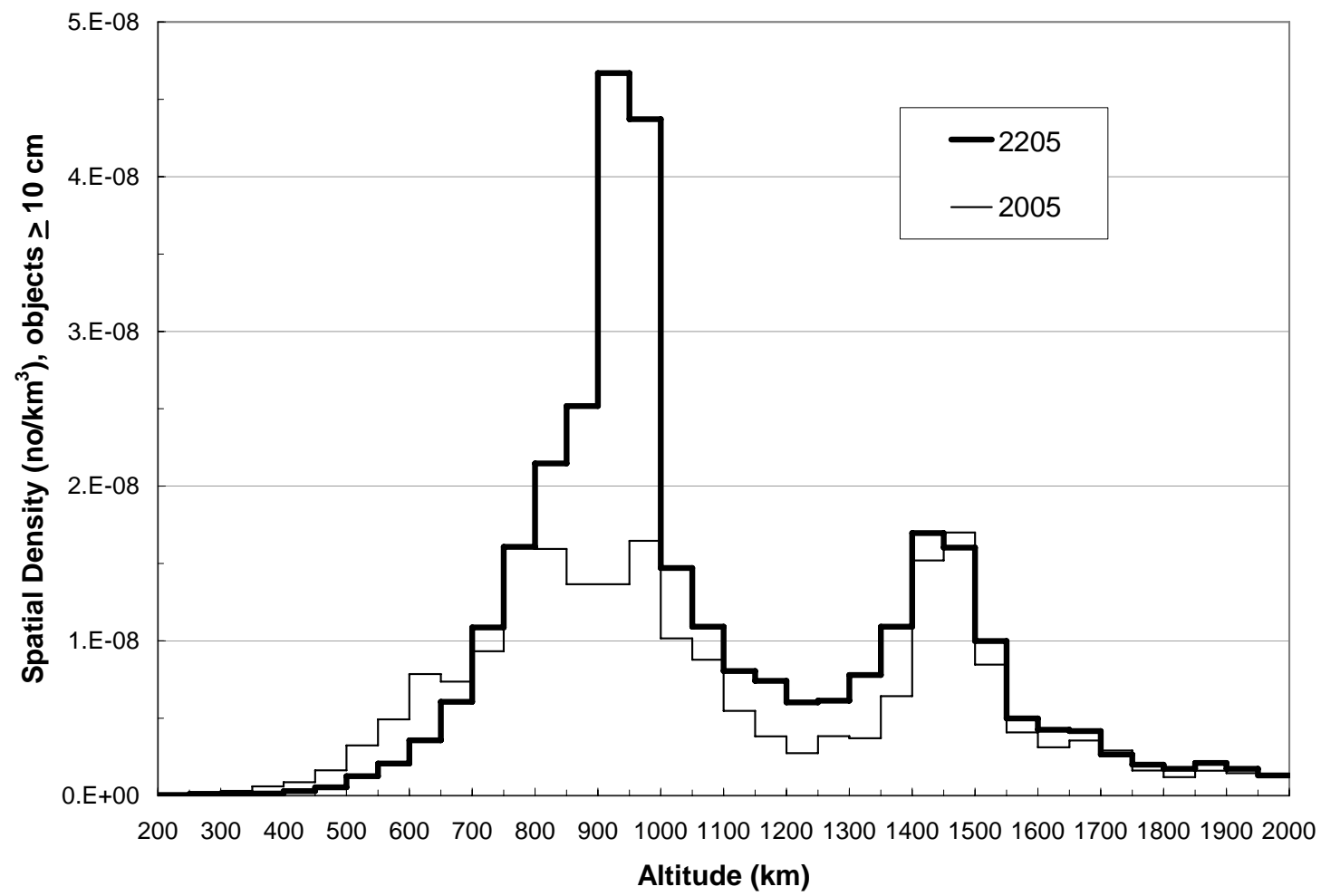




\section{Figure 8}

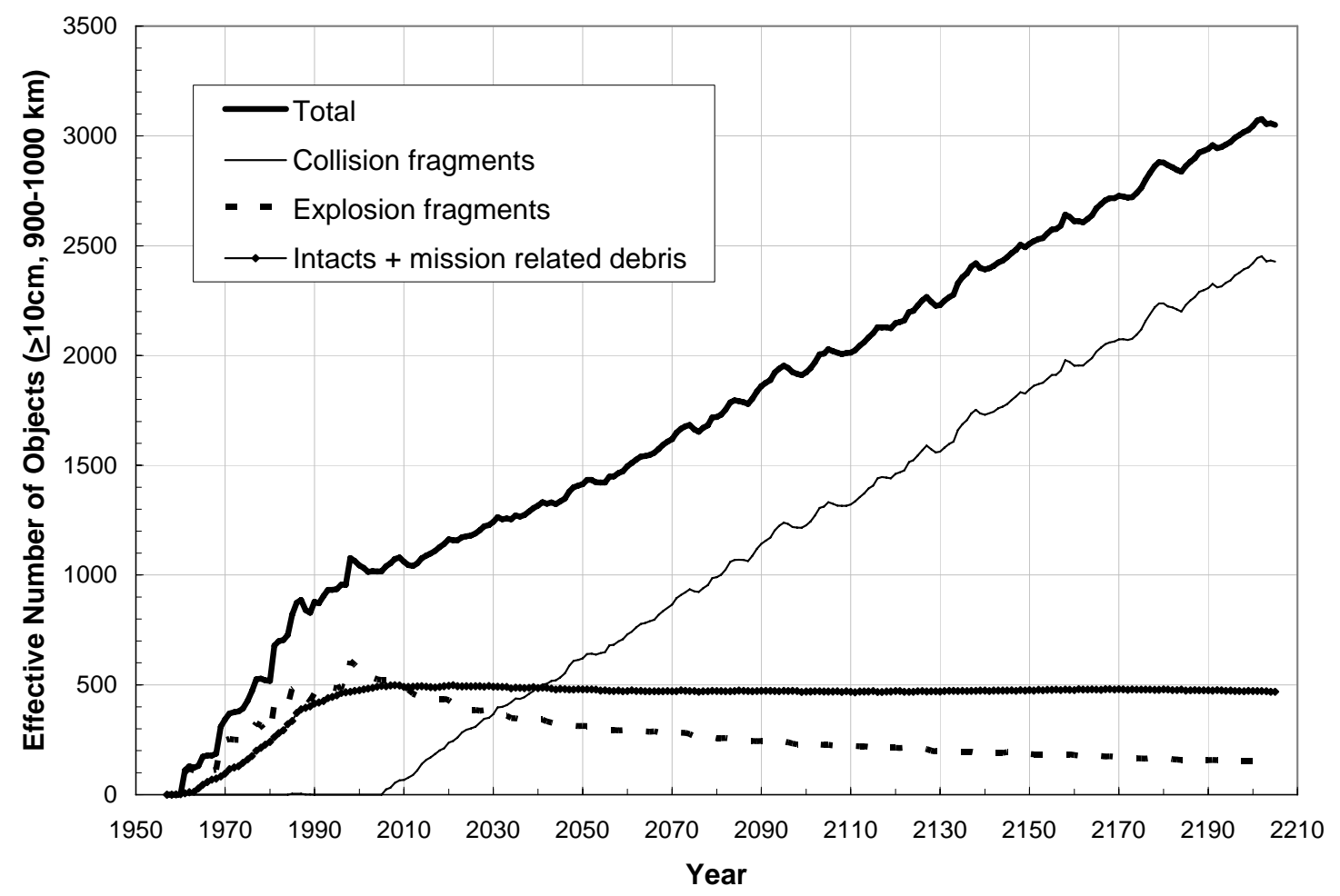

